\title{
Impact of siponimod on vaccination response in a randomized, placebo-controlled study
}

\section{OPEN}

Mike Ufer, MD, PhD

Kasra Shakeri-Nejad, MD

Anne Gardin, PhD

Zhenzhong $\mathrm{Su}, \mathrm{PhD}$

Ines Paule, $\mathrm{PhD}$

Thomas C. Marbury, MD

Eric Legangneux, MD

Correspondence to

Dr. Ufer:

mikeufer@gmx.de

\section{ABSTRACT}

Objective: To evaluate effects of siponimod on response to T-cell-dependent (influenza) and Tcell-independent (pneumococcal polysaccharide vaccine [PPV-23]) vaccinations in healthy participants.

Methods: In this double-blind, placebo-controlled, parallel-group study, each participant underwent a 7-week treatment period and received intramuscular injections of influenza and PPV-23 vaccines (day 21). Participants were randomized to 4 treatment groups $(\mathrm{N}=$ 30 each) and received placebo or siponimod $2 \mathrm{mg}$ once daily in concomitant, interrupted, or preceding fashion. Individual response to vaccination was defined by a $\geq 4$-fold (influenza) antibody titer increase and by a $\geq 2$-fold increase in serotype-specific immunoglobulin (lg) $G$ concentrations (PPV-23) on day 28 vs baseline. Responder rates were compared using noninferiority analysis.

Results: Mean influenza titers were similar to placebo in the preceding and interrupted groups but lower in the concomitant group. The proportion of participants with influenza titers $\geq 40$ four weeks after vaccination (seroprotection) was similar to placebo across all groups and antigens. In each treatment group, response criteria were met for 3 of 4 antigens including H1N1 and H3N2. A noninferior response was determined in the context of preceding treatment but not interrupted or concomitant treatment. Regarding PPV-23, approximately $90 \%-100 \%$ of participants exhibited $a \geq 2$-fold increase in IgG concentrations vs baseline. Noninferior responder rates were determined for each siponimod treatment group.

Conclusions: Siponimod treatment had no relevant effect on antibody response to PPV-23. European Medicines Agency response criteria were essentially met for influenza, but titers were lower on concomitant treatment. Overall, these data suggest that siponimod has limited effect on the efficacy of vaccinations with neoantigens.

Classification of evidence: This study provides Class II evidence that in healthy persons, siponimod had limited effect on the immune response following influenza or pneumococcal vaccinations. Neurol Neuroimmunol Neuroinflamm 2017;4:e398; doi: 10.1212/NXI.0000000000000398

\section{GLOSSARY}

$\mathbf{A E}=$ adverse event; $\mathbf{C l}=$ confidence interval; EMA = European Medicines Agency; GMT = geometric mean titer; $\mathbf{H I A}=$ hemagglutination inhibition assay; $\mathbf{l g}=$ immunoglobulin; $\mathbf{L C}$-MS = liquid chromatography-mass spectrometry; $\mathbf{M S}=$ multiple sclerosis; PD = pharmacodynamic; PK = pharmacokinetic; PPV-23 = pneumococcal polysaccharide vaccine; $\mathbf{q} \cdot \mathbf{d}$. $=$ once daily; $\mathbf{S 1 P}=$ sphingosine 1-phosphate.

Siponimod (BAF312) is a sphingosine 1-phosphate (S1P) receptor modulator currently under clinical development for the treatment of secondary progressive multiple sclerosis (MS). ${ }^{1}$ It selectively binds to the receptor subtypes $1\left(\mathrm{~S}_{1} \mathrm{P}_{1}\right)$ and $5\left(\mathrm{~S}_{\mathrm{P}}\right)$ as an agonist and promotes prolonged receptor internalization (functional antagonism) preventing egression of autoreactive lymphocytes from lymph nodes. ${ }^{2,3}$

From the Novartis Pharma AG (M.U., K.S.-N., A.G., Z.S., I.P., E.L.), Basel, Switzerland; and Orlando Clinical Research Center (T.C.M.), Orlando, FL.

Funding information and disclosures are provided at the end of the article. Go to Neurology.org/nn for full disclosure forms. The Article Processing Charge was funded by the authors.

This is an open access article distributed under the terms of the Creative Commons Attribution-NonCommercial-NoDerivatives License 4.0 (CC BY-NC-ND), which permits downloading and sharing the work provided it is properly cited. The work cannot be changed in any way or used commercially without permission from the journal. 
As a result of its pharmacodynamic (PD) properties, siponimod dose dependently reduces the total lymphocyte count. ${ }^{1}$ This effect is reversible and also applicable to other S1P modulators. ${ }^{4-7}$ This reduction of lymphocyte count may lead to compromised immune response function and thus potentially limit the effectiveness of vaccinations that are eventually required in the target population. ${ }^{8,9}$ Given the shorter elimination half-life of siponimod as compared to the first-in-class S1P modulator fingolimod (Gilenya ${ }^{\circledR}$ ), a faster recovery of immune response function after treatment pause was to be expected. ${ }^{10}$

Therefore, this phase-I immune response study assessed not only the effects of concomitant administration but also of preceding and interrupted siponimod administration on vaccination efficacy. Influenza vaccine and pneumococcal polysaccharide vaccine (PPV-23; Pneumovax $\left.{ }^{\circledR}\right)$ served as T-cell-dependent and T-cell-independent antigen challenges, respectively.

METHODS Study design, drug administration, and vaccinations. This was a randomized, double-blind, singlecenter, placebo-controlled, parallel-group study. The primary research question was to assess whether and to what extent siponimod treatment may influence the efficacy of vaccinations with influenza and PPV-23. This study provides Class II evidence in healthy participants who were equally randomized to 4 different treatment groups (figure 1).

In group 1, the effect of concomitant (during vaccination) siponimod administration on vaccination response was investigated. In groups 2 and 3, the effect of preceding (until 7 days before vaccination) or interrupted (treatment pause from 10 days before until 14 days after vaccination) siponimod administration was assessed. These 2 groups have been included to identify whether a short-term treatment pause around the time of vaccination could effectively restore immune response function. Group 4 received placebo and served as a reference population. All 4 groups were synchronized with respect to the time of vaccination to maintain treatment blinding.

Siponimod was orally administered (by study staff) at a therapeutic dose of $2 \mathrm{mg}$ once daily (q.d.), preceded by a 5-day uptitration period to prevent any bradyarrhythmic event. ${ }^{11}$ Influenza vaccine (Fluvirin ${ }^{\circledR}$ ) and PPV-23 (Pneumovax ${ }^{\circledR}$ ) were selected as

Figure 1 Study design

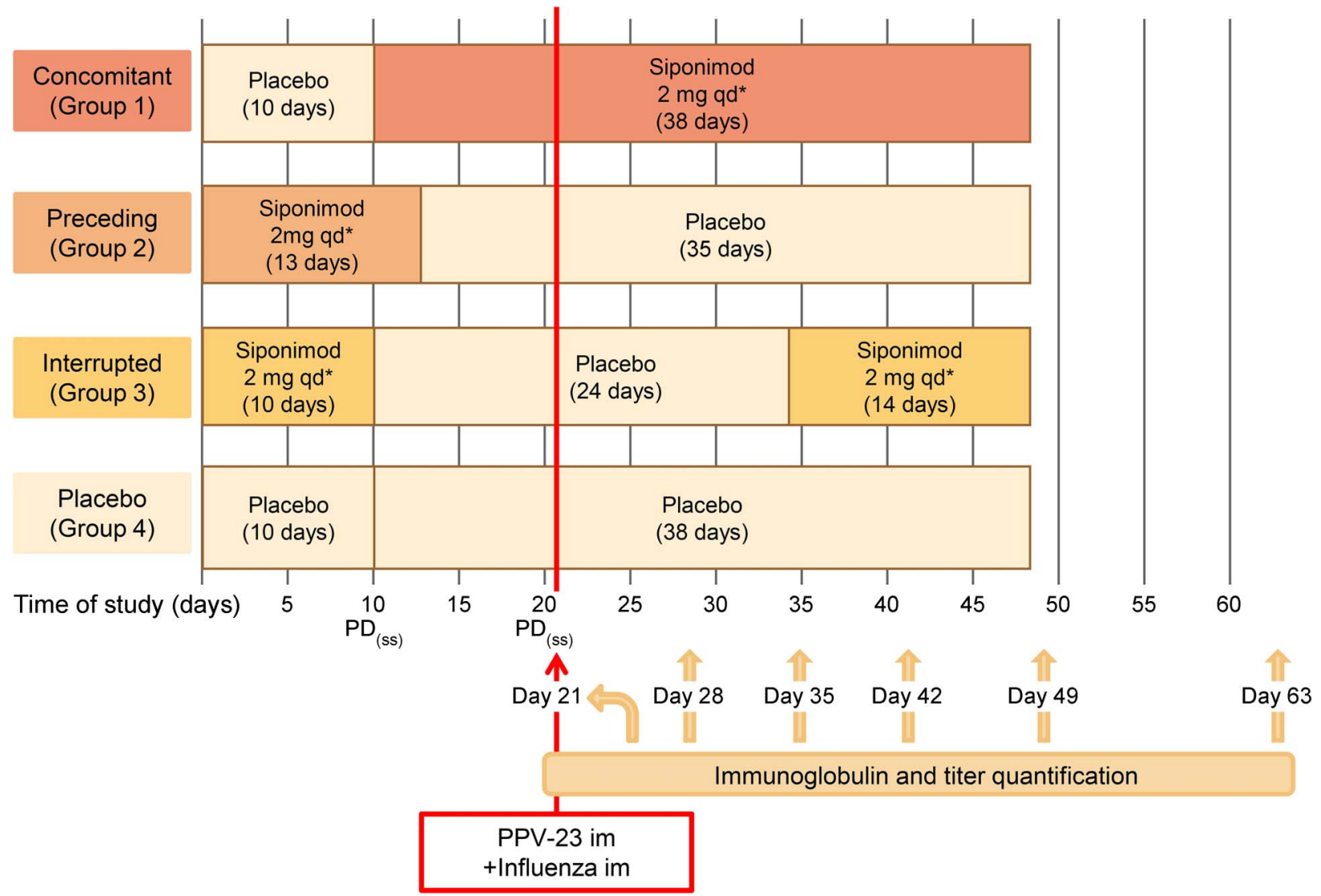

The study comprised a 28-day screening period, a 48-day treatment period, and a 14-day follow-up period. *Siponimod treatment was initiated according to a 5 -day up-titration scheme, beginning with $0.25 \mathrm{mg}$ on days $1-2$ followed by $0.5 \mathrm{mg}$ on day $3,0.75 \mathrm{mg}$ on day $4,1.25 \mathrm{mg}$ on day 5 , and $2 \mathrm{mg}$ on subsequent days. $\mathrm{im}=$ intramuscular; $\mathrm{PD}(\mathrm{ss})=$ pharmacodynamics at steady state; q.d. = once daily. 
T-cell-dependent and T-cell-independent antigen challenges, respectively.

A quadrivalent influenza vaccine recommended for use in North America during the 2014/2015 season was used. It contained 4 different antigens, namely the influenza "A"-antigens influenza-A/California/7/2009 (H1N1) and influenza-A/Texas/ $50 / 2012(\mathrm{H} 3 \mathrm{~N} 2)$ as well as the "B" antigens influenza-B/ Brisbane/60/2008 (B-Bri) and influenza-B/Massachusetts/2/ 2012 (B-Mas).

The drug administration period spanned over 7 weeks (study days 1-49). No medication other than the study drug was allowed from 4 weeks before initial dosing until study day 63 unless required for the treatment of adverse events (AEs).

On study day 21 , each participant received both vaccines intramuscularly. Antibody concentrations and titers were determined just before vaccination (baseline) as well as 7, 14, 21, 28 , and 42 days after vaccination. Accordingly, blood samples for antibody quantification were collected on study days 21 , $28,35,42,49$, and 63 by direct venipuncture or via an indwelling cannula inserted into a forearm vein.

Hemagglutination inhibition assay (HIA) was used to detect strain-specific anti-influenza antibodies, applying serial 2-fold dilutions of serum. HIA titers corresponded to the highest dilution of serum that achieved complete inhibition of hemagglutination. In regard to PPV-23, immunoglobulin (Ig) M and IgG concentrations were determined.

Standard protocol approvals, registrations, and participant consents. The study protocol was approved by the local ethics committee, and the study was conducted in accordance with the Declaration of Helsinki and Good Clinical Practice guidelines. ${ }^{12,13}$ Written informed consent was obtained from each participant before enrollment.
Study population. Healthy men or women of nonchildbearing potential were enrolled (age: 18-55 years; body weight: $\geq 50 \mathrm{~kg}$; body mass index: $18-30 \mathrm{~kg} / \mathrm{m}^{2}$ ). Absence of any clinically relevant disease and normal laboratory, vital sign, and ECG data were required, along with a total white blood cell count or lymphocyte count within 1.5 -fold of the normal range.

Participants were not eligible if they had received any previous vaccination with PPV-23 or influenza or any vaccination with live-attenuated vaccines within 2 months before enrollment. Each study participant was randomly assigned to any of the 4 different treatment groups in a 1:1:1:1 ratio based on their randomization number that was generated using a validated, computerized system and concealed from participants and study staff.

PD end points. Titer and antibody concentrations were quantified over time. Primary and secondary PD end points were determined by comparing the data obtained 4 weeks after vaccination (study day 49) with baseline data (study day 21).

Efficacy of influenza vaccination was evaluated in accordance with European Medicines Agency (EMA) guidelines ${ }^{14}$ that require at least one of the following 3 criteria to be met: (1) seroprotection rate $\geq 70 \%$ (i.e., proportion of participants achieving a postvaccination titer $\geq 40$ ); (2) postvaccination increase in geometric mean titers (GMTs) $\geq 2.5$ vs baseline; (3) seroresponse rate $\geq 40 \%$ (i.e., proportion of participants achieving seroconversion, i.e., $\geq 4$-fold titer increase post- vs pre-vaccination). In addition, responder analysis was performed with response defined as a $\geq 4$-fold increase in titers 4 weeks after vaccination compared with baseline (seroconversion). Finally, the proportion of seronegative participants was determined as defined by baseline titers $<1: 10$.

In regard to PPV-23, responder analysis was performed with response defined as a $\geq 2$-fold increase in IgG concentrations 4

Table 1 Participant demographics and disposition

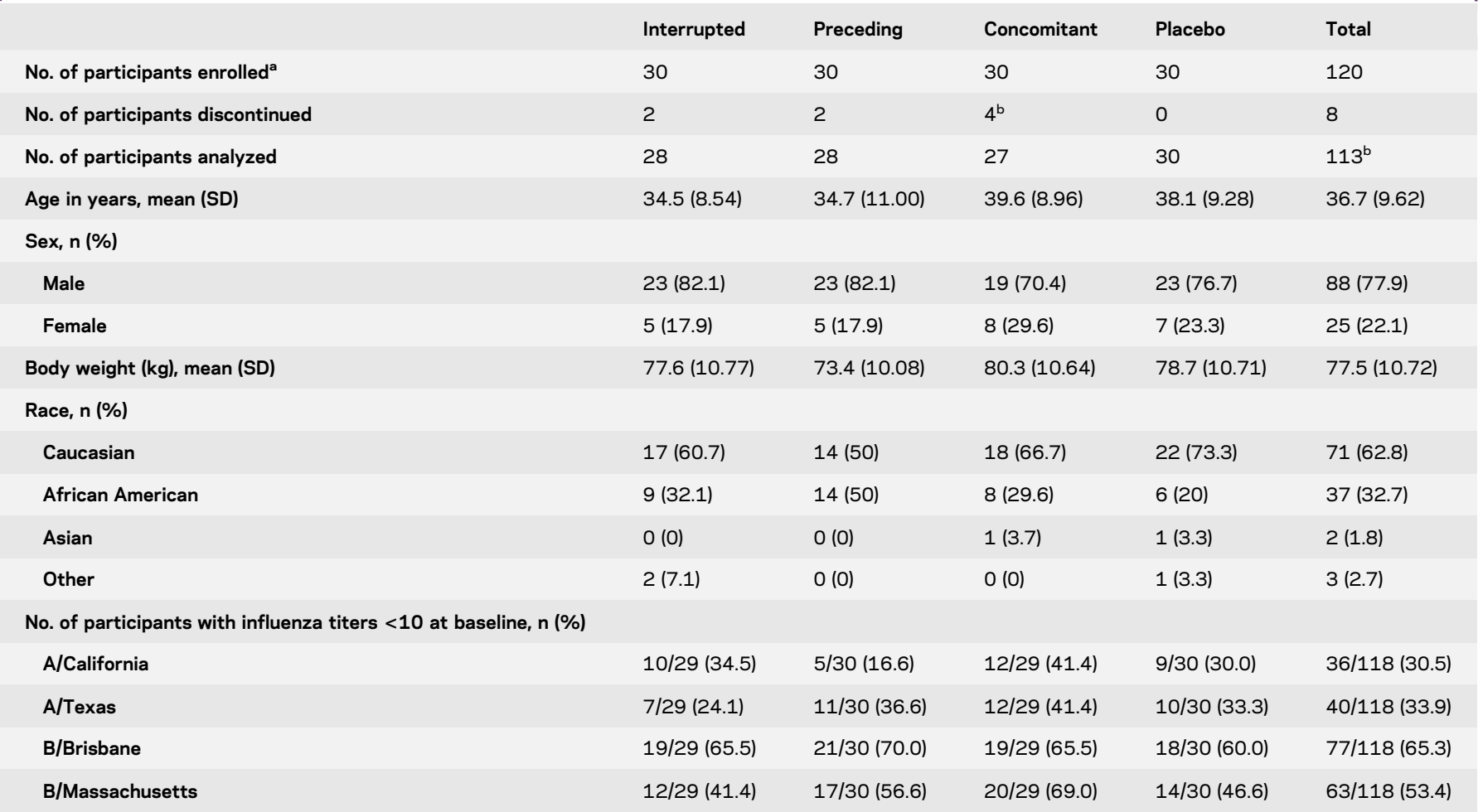

${ }^{\text {a Additional }} 16$ participants ( $n=4$ per group) had initially been enrolled but were discontinued before vaccination for administrative reasons.

${ }^{b}$ One of the discontinued participants was included in the PD analysis, as this participant was discontinued after study day 49. 
weeks after vaccination compared with baseline. ${ }^{15,16}$ In addition, IgM concentrations were determined to better characterize the time course of antibody response.

Pharmacokinetics and other assessments. Blood samples were repeatedly collected at 14 time points during the 7-week drug administration period (days 3, 6, 8, 10, 13, 16, 18, 21, $28,37,40,42,45$, and 49) as well as 2 weeks after the last dosing (day 63). Plasma concentrations of siponimod were measured using a validated LC-MS/MS method with a lower limit of quantification of $0.05 \mathrm{ng} / \mathrm{mL} .{ }^{17}$ Safety assessments included monitoring of AEs, ECG, laboratory, and vital sign data.

Statistical analyses. Efficacy analyses were conducted in all participants with antibody titer or concentration data available at least at baseline and 4 weeks after vaccination. The safety population comprised all participants exposed to at least a single dose of the study drug.

The proportion of participants who showed increased titers and $\operatorname{IgG}$ concentrations by $\geq 4$-fold (influenza) and $\geq 2$-fold (PPV-23) after vaccination was determined along with $95 \%$ confidence intervals (CIs) for the difference against placebo. For influenza, GMTs at baseline and day 28 and the respective ratios (post- vs pre-vaccination) were summarized by the strain and treatment group.

A noninferiority analysis was conducted to compare responder rates between each siponimod treatment group and placebo using a 1-sided $\chi^{2}$ test. Noninferiority was concluded if the lower limit of $95 \%$ CIs for difference of proportions was within the predefined noninferiority margin of 0.3 (i.e., $\leq 30 \%$ lower responder rates with siponimod vs placebo that was considered a clinically meaningful difference).

RESULTS Participant disposition and demographics. A total of 120 participants ( $\mathrm{N}=30$ per group) were enrolled. Of these, 112 (93.3\%) completed the study, whereas 8 participants discontinued treatment owing to administrative issues $(\mathrm{n}=5)$, AEs $(\mathrm{n}=2)$, or protocol deviation $(\mathrm{n}=1)$ (table 1$)$.

Demographic data including age, sex, body weight, and race were balanced across all treatment groups (table 1). The study population predominantly consisted of men $(77.9 \%)$, with an age range of $18-55$ years and a mean overall age of 36.7 years (SD, 9.6).

The proportion of participants with influenza titers $<10$ at baseline was essentially similar across all treatment groups with respect to each antigen (table 1).

PDs after influenza vaccination. GMTs over time indicate that peak antigen responses were reached on day

Figure 2 Antihemagglutinin inhibition titers (A) and immunoglobulin (Ig) concentrations (B) over time by the treatment group

A

A/California

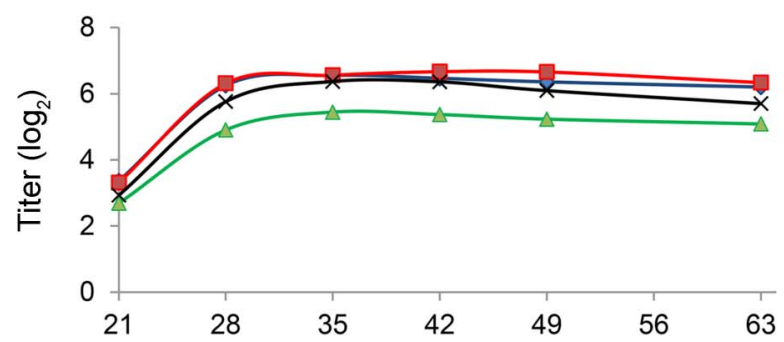

B/Brisbane

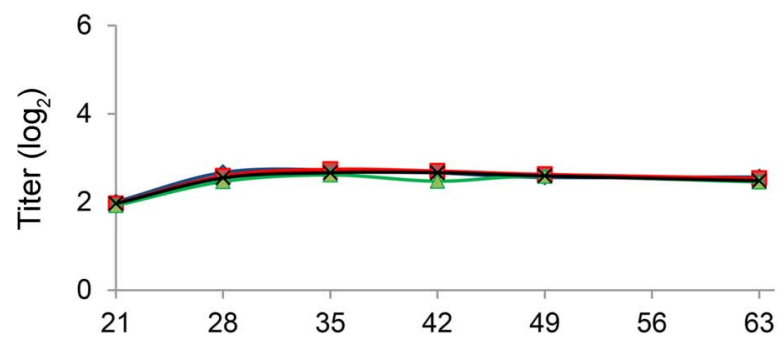

B

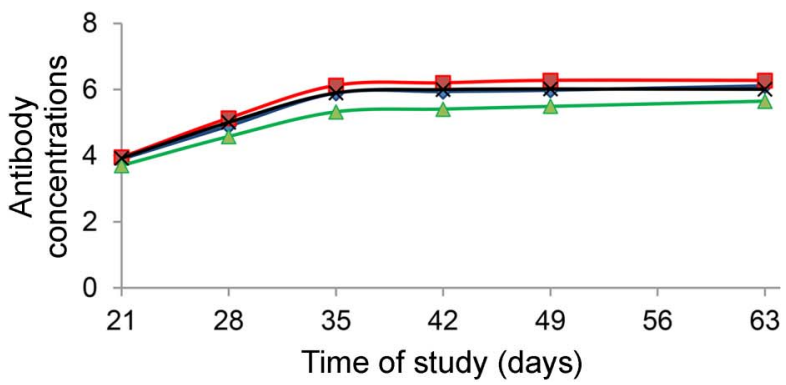

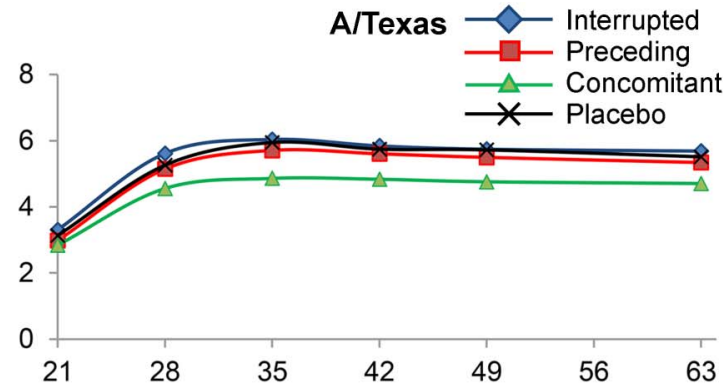

B/Massachusettes
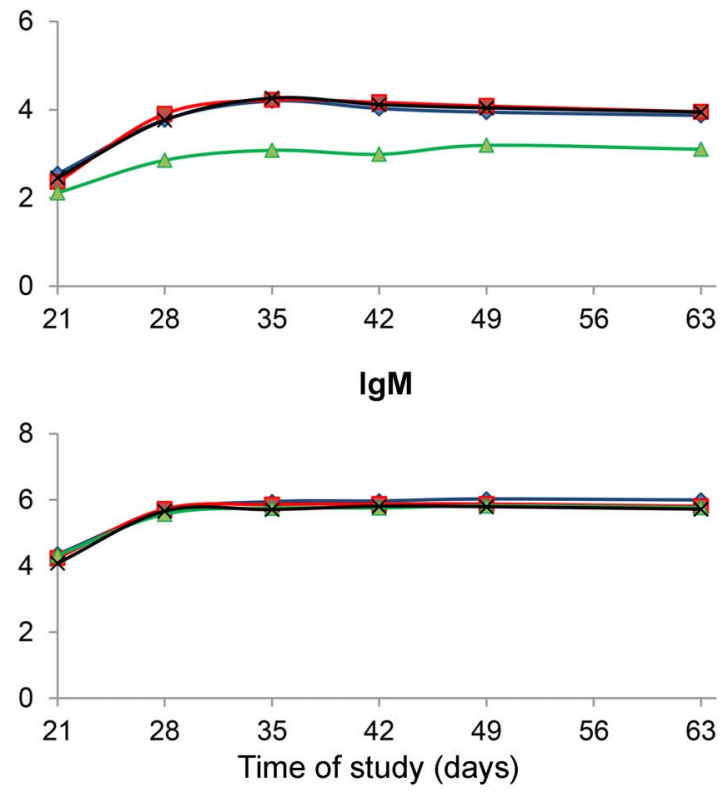

Logarithmic transformation of titer and IgG/IgM data was applied to the base 2. Error bars display SDs. 
28 after vaccination (figure 2). In regard to interrupted and preceding treatments, the titers over time were essentially equivalent to placebo. However, lower titers were determined for 3 of 4 antigens on concomitant treatment.

Influenza titer data have been evaluated with respect to each of the 3 EMA response criteria, namely seroprotection rate $\geq 70 \%$, GMT titer increase $\geq 2.5$ vs baseline, and seroresponse rate $\geq 40 \%$.

1. The proportion of participants with influenza titers $\geq 40$ at 4 weeks after vaccination (i.e., seroprotection) was essentially similar across all treatment groups with respect to each antigen (figure $3)$. In each treatment group, $\geq 70 \%$ of participants achieved seroprotection for both A-antigens California (H1N1) and Texas (H3N2), thereby meeting the respective EMA response criterion. The proportion of participants who achieved seroprotection in response to $\mathrm{B}$-antigens was much lower. In regard to B-Massachusetts, the response threshold of $\geq 70 \%$ participants achieving seroprotection was only met in the preceding and placebo groups, whereas in regard to B-Bri, this was not met in any treatment group.

2. In each treatment group, GMTs of both A-antigens California (H1N1) and Texas (H3N2) markedly increased, clearly exceeding the response threshold of $\geq 2.5$-fold, compared with baseline, as defined by EMA guidelines (table 2). ${ }^{14}$ However, the titer ratios were lower in the concomitant group as compared to the other treatment groups for both $\mathrm{H} 1 \mathrm{~N} 1$ (12.7 vs 19.9-28.3) and H3N2 (6.8 vs 11.5-13.3). In regard to B-antigens, the GMT ratios were much lower compared with A-antigens but essentially similar across all treatment groups.

3. Similar data were obtained with respect to the proportion of participants achieving seroconversion, i.e., $\geq 4$-fold increase in titer compared with baseline (table 2). For both A-antigens California (H1N1) and Texas (H3N2), this seroresponse rate clearly exceeded the EMA response threshold of $40 \%$ in each treatment group. In regard to B-antigens, seroresponse rates were generally lower but essentially similar across all treatment groups.

The responder analysis showed a noninferior proportion of participants achieving seroconversion with respect to each antigen in the preceding treatment group. However, noninferiority could not be statistically confirmed in the interrupted and concomitant treatment groups. Here, the proportion of participants achieving seroconversion was approximately $15 \%-30 \%$ lower compared with placebo.

PDs after PPV-23 vaccination. The time course profile indicates rising antibody concentrations until approximately 1 week (study day 28) for IgM and 2 weeks

Figure 3 Proportion of participants achieving seroprotection after influenza vaccination

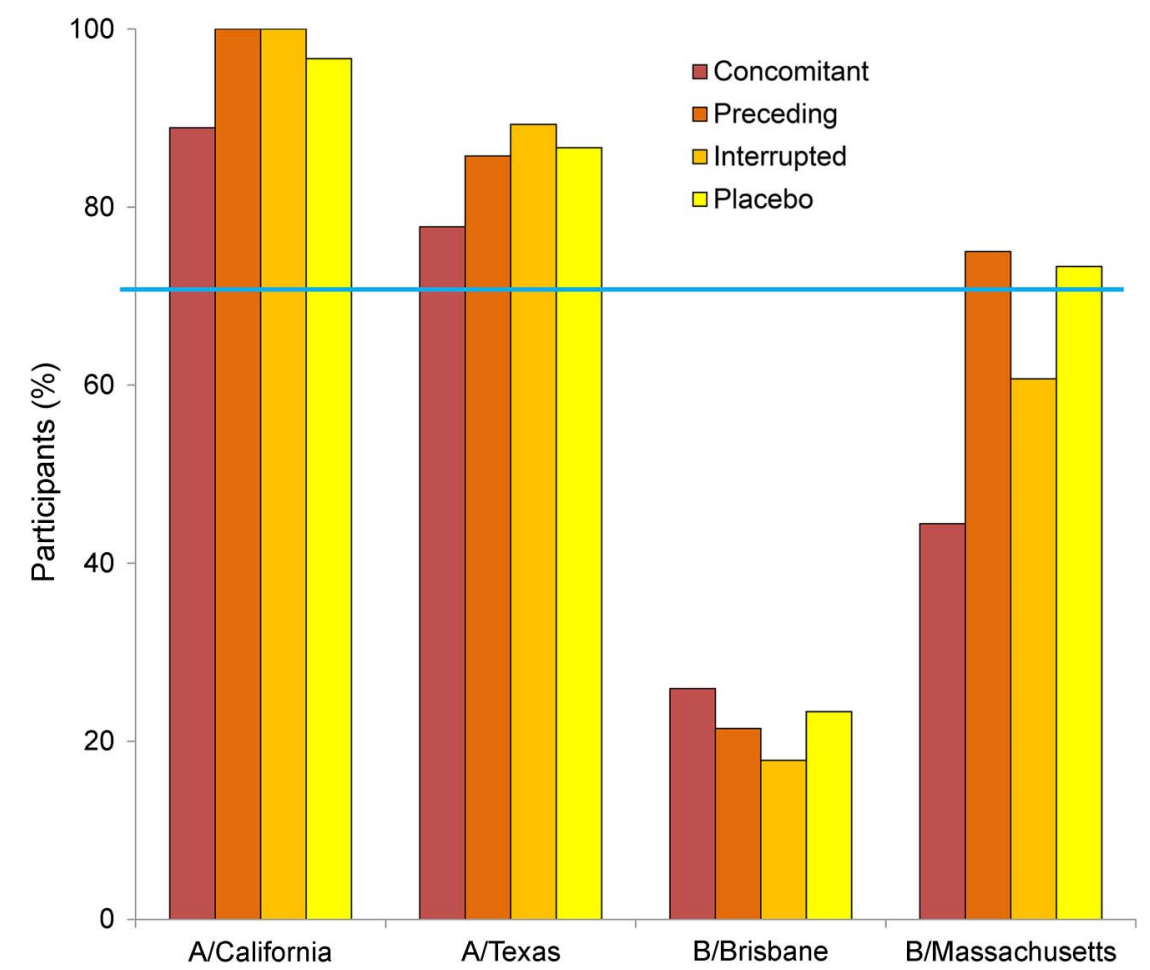

Seroprotection is defined by titers $\geq 40$ at day 28 after vaccination. Efficacy of influenza vaccination is achieved, provided that $\geq 70 \%$ of participants achieve seroprotection (as indicated by the blue line). 


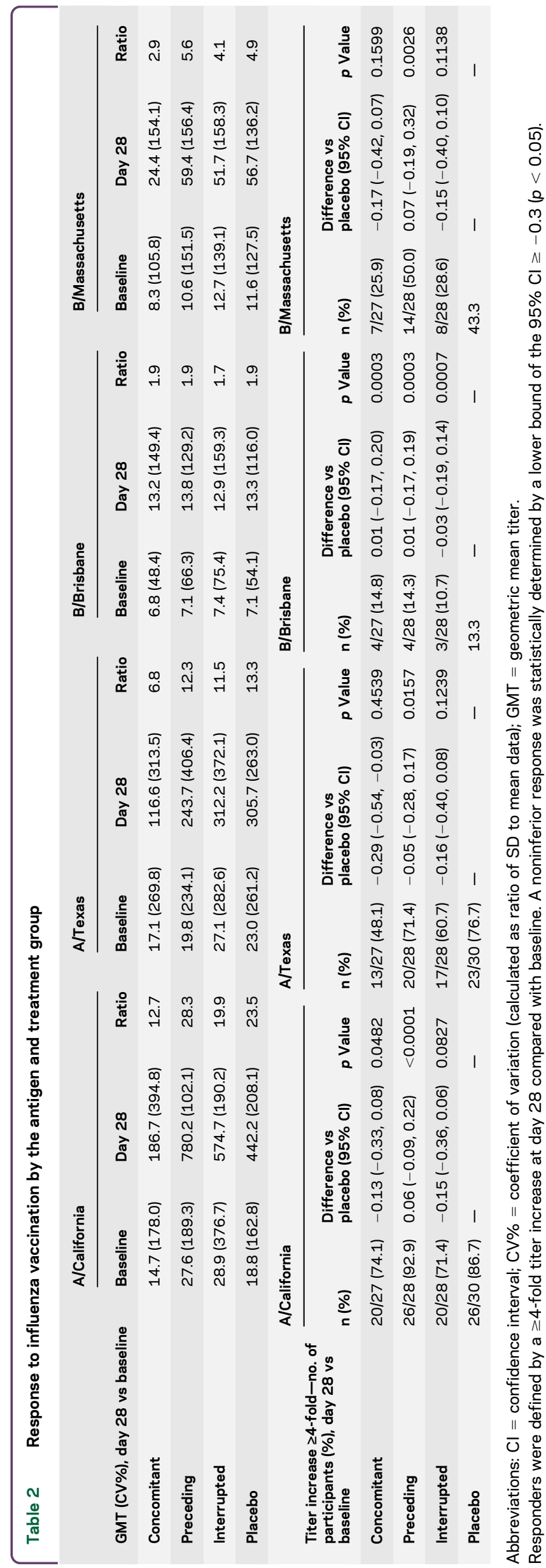

after vaccination (study day 35) for IgG (figure 2). IgG and IgM concentrations were highly similar across all treatment groups.

In each treatment group, approximately $90 \%-100 \%$ of participants showed a $\geq 2$-fold increase in $\mathrm{IgG}$ concentrations on day 28 after vaccination compared with baseline, thus meeting the predefined response threshold. Geometric mean ratios of $\mathrm{IgG}$ and IgM concentrations on day 28 after vaccination compared with baseline were also similar across all treatment groups (table 3).

Accordingly, the responder analysis revealed noninferior responder rates for each siponimod treatment group in comparison with placebo $(p<0.001$; table 3$)$.

Safety. Siponimod 2 mg q.d. was safe and well tolerated in healthy participants. This was primarily indicated by a similar incidence of AEs in the siponimod treatment groups and the placebo group $(35.3 \%-55.9 \%$ vs $38.3 \%)$. Most AEs were of mild intensity, had an early onset, and spontaneously resolved within 24 hours. The most commonly reported system organ class was nervous system disorders with headache representing the most common individual AE. Two participants discontinued the study because of AEs that were suspected to be study drug related (insomnia and increased transaminases). The $\mathrm{AE}$ of increased transaminases was asymptomatic and transient but was considered serious according to prespecified criteria (i.e., AST $>8$ fold the upper limit of normal). The participant admitted heavy exercise before the event, which was accompanied by increased creatine kinase concentrations. Laboratory, vital sign, and ECG data did not reveal any systematic trend over time or other clinically relevant findings. Moreover, there were no cases of clinically relevant bradycardia or atrioventricular blocks identified through online cardiac monitoring.

Pharmacokinetics. Repeated measurements of siponimod plasma concentrations during the 7-week treatment period indicated that all participants in each of the siponimod treatment groups were adequately exposed. As expected, in most individuals, steady-state conditions were achieved after 10 days of treatment, with similar plasma concentrations in each treatment group noted on the last day of siponimod administration (group 1: $16.4 \mathrm{ng} / \mathrm{mL}$; group 2: 21.2 $\mathrm{ng} / \mathrm{mL}$; group 3: $19.5 \mathrm{mg} / \mathrm{mL}$; and group 4: $17.4 \mathrm{ng} /$ $\mathrm{mL}$ ). Moreover, the concentration-time profile was in line with the respective dosing scheme in each group (data not shown).

DISCUSSION The study results suggest only a minor effect of siponimod treatment on the efficacy of influenza and PPV-23 vaccinations primarily evidenced by 
Table 3 Response to PPV-23 vaccination by the immunoglobulin and treatment group

\begin{tabular}{|c|c|c|c|c|c|c|c|c|}
\hline \multicolumn{2}{|c|}{ Geometric mean (CV\%), day 28 vs baseline } & \multicolumn{3}{|c|}{$\lg G$} & & \multicolumn{3}{|c|}{$\lg M$} \\
\hline \multicolumn{2}{|l|}{ Concomitant } & $40.4(121.1)$ & $242.8(87.8)$ & 6.0 & & $75.8(64.1)$ & $335.2(55.4)$ & 4.4 \\
\hline \multicolumn{2}{|l|}{ Preceding } & $51.9(81.5)$ & $532.0(95.1)$ & 10.2 & & $69.8(69.1)$ & $355.6(75.1)$ & 5.1 \\
\hline \multicolumn{2}{|l|}{ Interrupted } & $49.2(81.9)$ & $393.8(105.4)$ & 8.0 & & $77.6(61.5)$ & $412.6(97.2)$ & 5.3 \\
\hline \multicolumn{2}{|l|}{ Placebo } & $50.7(89.6)$ & 411.1 (147.2) & 8.1 & & $59.3(111.0)$ & 326.7 (87.9) & 5.5 \\
\hline $\begin{array}{l}\text { Ig concentration increase } \geq 2 \text {-fold, } \\
\text { day } 28 \text { vs baseline }\end{array}$ & N (\%) & \multicolumn{2}{|c|}{ Difference vs placebo $(95 \% \mathrm{Cl})$} & & $p$ Value & $\mathrm{N}(\%)$ & $95 \% \mathrm{Cl}$ & $p$ Value \\
\hline Concomitant & $27 / 27(100)$ & \multicolumn{2}{|c|}{$0.07(-0.03$ to 0.16$)$} & & $<0.0001$ & NA & NA & - \\
\hline Preceding & $28 / 28(100)$ & \multicolumn{2}{|c|}{$0.07(-0.03$ to 0.16$)$} & & $<0.0001$ & NA & NA & - \\
\hline Interrupted & $25 / 28(89.3)$ & \multicolumn{2}{|c|}{$-0.04(-0.19$ to 0.10$)$} & & 0.0002 & NA & NA & - \\
\hline
\end{tabular}

Abbreviations: $\mathrm{Cl}=$ confidence interval; $\mathrm{CV} \%$ = coefficient of variation (calculated as ratio of SD to mean data); lg = immunoglobulin; NA = not applicable; PPV-23 = pneumococcal polysaccharide vaccine.

Responders were defined by a $\geq 2$-fold increase in lgG concentrations at day 28 compared with baseline. A noninferior response was statistically determined by a lower bound of the $95 \% \mathrm{Cl} \geq-0.3(p<0.05)$.

Responder analyses were performed by comparing proportion differences in each active treatment group against placebo using a noninferiority margin of 0.3 .

Response was a priori defined by a $\geq 2$-fold increase in IgG concentrations. Hence, this responder analysis was not applicable for lgM data.

(1) $\geq 70 \%$ of participants achieving seroprotection (i.e., titers $\geq 40$ on day 28 ) in each treatment group with respect to H1N1 and H3N2 (figure 3) ${ }^{14}$ and (2) approximately $90 \%-100 \%$ responders to PPV-23 vaccination (i.e., $\geq 2$-fold increase in $\mathrm{IgG}$ concentrations on day 28 vs baseline) in each treatment group (table 3). Vaccinations with influenza and PPV-23 have been used as a model of T-celldependent and T-cell-independent antigen challenge in multiple immune response studies, although there is evidence suggesting that PPV-23 may not act fully independent of T-cell function. ${ }^{18}$

Because influenza and pneumococcal infections may trigger serious consequences in the target population of patients with MS, ${ }^{19,20}$ results of this study are highly relevant for health care providers. To derive practical recommendations for clinical management, this study not only investigated the effect of concomitant siponimod treatment on vaccination response, as typically done in immune response studies, but also aimed to identify whether a short-term treatment pause around the time of vaccination could effectively restore immune response function. Therefore, 2 additional schedules were assessed, namely preceding and interrupted siponimod treatment. According to pharmacokinetic (PK)/PD modeling-based simulations, it has been predicted that after siponimod discontinuation, it takes approximately 7-10 days to reach $90 \%-95 \%$ of the baseline lymphocyte count (Novartis data on file). Therefore, siponimod treatment was terminated 7 and 10 days before the vaccination challenge in the preceding and interrupted treatment groups, respectively. In the interrupted treatment group, the potential effect of re-introducing siponimod treatment while antibody response mounted was investigated as well (i.e., 14 days after vaccination).

In the case of PPV-23, immunomodulatory therapies used for the treatment of inflammatory bowel diseases have affected vaccination efficacy in earlier clinical trials including the cytotoxic immunosuppressants 6-mercaptopurine, azathioprine, and methotrexate. ${ }^{15,16}$ However, our study results indicate that siponimod treatment has no clinically relevant effect on PPV-23 antibody response as evidenced by $\geq 90 \%$ of participants achieving the predefined response threshold in each treatment group. The response definition of $a \geq 2$-fold increase in IgG concentrations has also been applied in previous immune response studies. ${ }^{16,17}$

A positive influenza vaccination response according to EMA guidelines was confirmed if at least 1 of 3 criteria was met, namely seroprotection rate $\geq 70 \%$, GMT ratio $\geq 2.5$, or seroresponse rate $\geq 40 \% .{ }^{15}$ In this study, such positive response was determined in each treatment group for 3 of 4 antigens (H1N1, H3N2, and B-Massachusetts). Hence, no treatment effect was apparent when applying this response definition given equivalent results in each siponimod treatment group as in the placebo group. However, GMTs over time were lower on concomitant siponimod treatment. Accordingly, approximately 2 -fold lower ratios of titers on day 28 vs baseline were determined.

In regard to clinical relevance, influenza A strains (H1N1 and H3N2) are responsible for two-thirds 
of annual influenza infections, and thus, corresponding antibodies are often considered to be of greater importance for the prevention of influenza. ${ }^{21,22}$ In this study, influenza titers of antibodies directed against the A-antigens were higher than those of the $\mathrm{B}$-antigens in each treatment group. The proportion of responders (i.e., seroconversion rates) was also higher for the A-antigens (app. 70\%-90\% for A-Cal and $50 \%-70 \%$ for A-Tex) than that for the B-antigens (10\%-15\% for B-Bri and 25\%-50\% for B-Mas), the latter antigens generally being perceived as less clinically relevant. ${ }^{21,22}$

Study validity was determined based on PK and PD data obtained. In regard to PKs, the dose-exposure relationship was in line with previous data in healthy participants, and the concentration-time profile followed the dosing regimen in each group. ${ }^{1,23}$ In regard to PDs, the influenza seroconversion rates obtained in the placebo group were in line with expectations based on recently published reference data for this quadrivalent vaccine 2014/2015. ${ }^{24}$ Although participants were required to be vaccine naive, a majority of participants had influenza titers $\geq 10$ at baseline, which is not unexpected and may be attributed to previous antigen exposure. ${ }^{25}$ It is important that the proportion of participants with influenza titers $<10$ vs those with titers $\geq 10$ was similar across all treatment groups, indicating the absence of any study bias in this respect. Moreover, the fold changes in PPV-23 antibody concentrations were similar to data from previous studies. ${ }^{26,27}$

Similar immune response studies with other drugs approved for MS treatment have been conducted. ${ }^{28-30}$ Concomitant intake of the S1P modulator fingolimod was associated with lower keyhole limpet hemocyanin and PPV-23 antibody concentrations compared with placebo in healthy participants. ${ }^{29}$ This is in contrast to the results of this study, which indicated no clinically relevant effects of siponimod on response to PPV-23 vaccination irrespective of the treatment regimen administered. In fingolimod-treated patients with MS, lower response rates to influenza vaccination and tetanus toxoid have been determined. ${ }^{29}$ Overall, influenza titers were approximately 2 -fold lower on concomitant fingolimod treatment, which is essentially similar to siponimod data obtained with the concomitant group in this study. ${ }^{29}$ In another immune response study with the pyrimidine synthesis inhibitor teriflunomide (Aubagio $\left.{ }^{\circledR}\right)$, patients with relapsing MS showed adequate immune responses to seasonal influenza vaccination with teriflunomide treatment. ${ }^{30}$

As applicable to any phase-I immune response study, the ultimate clinical relevance of the influenza titer data and their effect on the risk of influenza infection cannot be definitively concluded, although an inverse correlation between influenza titers and infection rates has been determined. ${ }^{31}$ Yet, the quantitative relationship between PDs (i.e., titer levels) and efficacy (i.e., infection rates) is not very well characterized and varies also from year to year depending on vaccine composition. This makes it difficult to judge the appropriateness of the predefined noninferiority margin of $30 \%$. There is a general consensus though that titers $\geq 1: 40$ are considered as seroprotective because these confer to a $\geq 50 \%$ lower incidence of influenza infection and to a lower likelihood of a serious infection. ${ }^{15}$ In this study, GMTs at 4 weeks after influenza vaccination were considerably greater than 1:40 in all 3 siponimod treatment groups with respect to $\mathrm{H} 1 \mathrm{~N} 1$ and $\mathrm{H} 3 \mathrm{~N} 2$.

Finally, siponimod 2 mg q.d. was well tolerated in this study, as evidenced by a similar incidence of AEs in all siponimod as well as the placebo groups. The $\mathrm{AE}$ profile, with nervous system disorders and headache being the most predominant ones, was in line with data from other phase I studies conducted in healthy participants. ${ }^{1}$

In regard to clinical implications, this may be continued without changes in the context of PPV-23 vaccination, given the essentially unchanged antibody response to PPV-23 in the context of concomitant siponimod treatment. Siponimod treatment may also be continued at the time of influenza vaccination, but lower titers cannot be excluded in such case. If a placebo-like titer response is considered to be required, short-term treatment pauses before and after vaccination may be applied. The benefit of an unaffected titer response should be balanced against the medical need for continuous siponimod treatment on a case-by-case basis by the prescriber, depending on the risk of relapse or worsening of MS vs that of influenza infection.

Overall, the results of this study suggest that siponimod has only limited effect on the efficacy of vaccinations with neoantigens.

\section{AUTHOR CONTRIBUTIONS}

M.U. and K.S.-N.: study design/conceptualization, data analysis/interpretation, and manuscript writing. A.G., I.P., and E.L.: study design/conceptualization, data analysis/interpretation, and manuscript review. Z.S.: statistical analysis and manuscript review. T.C.M. (principal investigator): study conduct and manuscript review.

\section{ACKNOWLEDGMENT}

The authors thank Sivaram Vedantam (Novartis, Medical Communications) for technical editing support. They also thank Dr. Arnold S. Monto (University of Michigan, Ann Arbor, MI, USA) for expert advice on data analysis and interpretation. In addition, they also acknowledge the contributions of Beatriz Restrepo (SeaView Research, Inc., Miami, FL, USA) as coinvestigator.

\section{STUDY FUNDING}

This study was funded by Novartis Pharma AG, Basel, Switzerland.

\section{DISCLOSURE}

M. Ufer, Z. Su, I. Paule, and E. Legangneux are employed by and hold stock options in Novartis Pharma. K. Shakeri-Nejad is employed by 
Novartis Institutes for Biomedical Research. A. Gardin is employed by Novartis Pharma. T.C. Marbury is employed by and is an equity owner of Orlando Clinical Research Center. Go to Neurology.org/nn for full disclosure forms.

Received March 11, 2017. Accepted in final form June 23, 2017.

\section{REFERENCES}

1. Gentili A, Musella A, Bullitta S, et al. Siponimod (BAF312) prevents synaptic neurodegeneration in experimental multiple sclerosis. J Neuroinflammation 2016;13:207.

2. Gergely P, Nuesslein-Hildesheim B, Guerini D, et al. The selective sphingosine 1-phosphate receptor modulator BAF312 redirects lymphocyte distribution and has species-specific effects on heart rate. Br J Pharmacol 2012;167:1035-1047.

3. Matloubian M, Lo CG, Cinamon G, et al. Lymphocyte egress from thymus and peripheral lymphoid organs is dependent on S1P receptor 1. Nature 2004;427:355-360.

4. Brossard P, Derendorf $\mathrm{H}, \mathrm{Xu}$ J, Maatouk $\mathrm{H}$, Halabi A, Dingemanse J. Pharmacokinetics and pharmacodynamics of ponesimod, a selective S1P1 receptor modulator, in the firstin-human study. Br J Clin Pharmacol 2013;76:888-896.

5. Timony G, Brooks J, Hartung J, et al. Pharmacokinetics and pharmacodynamics of RPC1063 and its metabolites in healthy adult volunteers. Neurology 2014;82:P1.211.

6. Krosser S, Wolna P, Fischer TZ, et al. Effect of ceralifimod (ONO-4641) on lymphocytes and cardiac function: randomized, double-blind, placebo-controlled trial with an open-label fingolimod arm. J Clin Pharmacol 2015;55:1051-1060.

7. Mehling M, Hilbert P, Fritz S, et al. Antigen-specific adaptive immune responses in fingolimod-treated multiple sclerosis patients. Ann Neurol 2011;69:408-413.

8. Loebermann M, Winkelmann A, Hartung HP, Hengel H, Reisinger EC, Zettl UK. Vaccination against infection in patients with multiple sclerosis. Nat Rev Neurol 2011;8: 143-151.

9. Williamson EM, Berger JR. Central nervous system infections with immunomodulatory therapies. Continuum (Minneap Minn) 2015;21:1577-1598.

10. Juif PE, Kraehenbuehl S, Dingemanse J. Clinical pharmacology, efficacy, and safety aspects of sphingosine-1phosphate receptor modulators. Expert Opin Drug Metab Toxicol 2016;12:879-895.

11. Legangneux E, Gardin A, Johns D. Dose titration of BAF312 attenuates the initial heart rate reducing effect in healthy subjects. Br J Clin Pharmacol 2013;75:831-841.

12. Declaration of Helsinki-ethical principles for medical research involving human subjects. World Medical Association. Available at: http://sites.jamanetwork.com/declarationof-helsinki/index.html. Accessed April 20, 2017.

13. ICH harmonised tripartite guideline: guideline for good clinical practice E6(R1). International Conference on Harmonisation. Available at: http://www.ich.org/products/ guidelines.html. Accessed April 20, 2017.

14. European Agency for the Evaluation of Medicinal Products. Harmonisation of Requirements for Influenza Vaccines (CPMP/BWP/214/96): Note for Guidance on Harmonization of Requirements for Influenza Vaccines. London, United Kingdom; 1997.

15. Melmed GY, Agarwal N, Frenck RW, et al. Immunosuppression impairs response to pneumococcal polysaccharide vaccination in patients with inflammatory bowel disease. Am J Gastroenterol 2010;105:148-154.
16. Fiorino G, Peyrin-Biroulet L, Naccarato P, et al. Effects of immunosuppression on immune response to pneumococcal vaccine in inflammatory bowel disease: a prospective study. Inflamm Bowel Dis 2012;18:1042-1047.

17. Shakeri-Nejad K, Aslanis V, Veldandi U, et al. Pharmacokinetics, safety, and tolerability of siponimod (BAF312) in subjects with different levels of hepatic impairment: a single-dose, open-label, parallel-group study. Int J Clin Pharmacol Ther 2017;55:41-53.

18. Kobrynski LJ, Sousa AO, Nahmias AJ, Lee FK. Cutting edge: antibody production to pneumococcal polysaccharides required $\mathrm{CD} 1$ molecules and $\mathrm{CD} 8+\mathrm{T}$ cells. J Immunol 2005;174:1787-1790.

19. Confavreux C, Suissa S, Saddier P, Bourdes V, Vukusic S; Vaccines in Multiple Sclerosis Study G. Vaccinations and the risk of relapse in multiple sclerosis. Vaccines in multiple sclerosis study group. N Engl J Med 2001;344:319-326.

20. De Keyser J, Zwanikken C, Boon M. Effects of influenza vaccination and influenza illness on exacerbations in multiple sclerosis. J Neurol Sci 1998;159:51-53.

21. Balasingam S, Wilder-Smith A. Randomized controlled trials for influenza drugs and vaccines: a review of controlled human infection studies. Int J Infect Dis 2016;49:18-29.

22. Baxter D. Evaluating the case for trivalent or quadrivalent influenza vaccines. Hum Vaccin Immunother 2016;12: 2712-2717.

23. Biswal S, Polus F, Pal P, et al. Pharmacokinetic and pharmacodynamic interaction of siponimod (BAF312) and propranolol in healthy subjects. Int J Clin Pharmacol Ther 2015;53:855-865.

24. WHO. Recommended composition of influenza virus vaccines for use in the 2014-2015 northern hemisphere influenza season. 2014. Available at: http://www.who.int/ influenza/vaccines/virus/recommendations/2014_15_ north/en/. Accessed April 20, 2017.

25. Hancock K, Veguilla V, Lu X, et al. Cross-reactive antibody responses to the 2009 pandemic H1N1 influenza virus. N Engl J Med 2009;361:1945-1952.

26. Chioato A, Noseda E, Felix SD, et al. Influenza and meningococcal vaccinations are effective in healthy subjects treated with the interleukin-1 beta-blocking antibody canakinumab: results of an open-label, parallel group, randomized, singlecenter study. Clin Vaccin Immunol 2010;17:1952-1957.

27. Chioato A, Noseda E, Stevens M, Gaitatzis N, Kleinschmidt A, Picaud H. Treatment with the interleukin-17A-blocking antibody secukinumab does not interfere with the efficacy of influenza and meningococcal vaccinations in healthy subjects: results of an open-label, parallel-group, randomized single-center study. Clin Vaccin Immunol 2012;19: 1597-1602.

28. Boulton C, Meiser K, David OJ, Schmouder R. Pharmacodynamic effects of steady-state fingolimod on antibody response in healthy volunteers: a 4-week, randomized, placebo-controlled, parallel-group, multiple-dose study. J Clin Pharmacol 2012;52:1879-1890.

29. Kappos L, Mehling M, Arroyo R, et al. Randomized trial of vaccination in fingolimod-treated patients with multiple sclerosis. Neurology 2015;84:872-879.

30. Bar-Or A, Freedman MS, Kremenchutzky M, et al. Teriflunomide effect on immune response to influenza vaccine in patients with multiple sclerosis. Neurology 2013;81:552-558.

31. Ohmit SE, Petrie JG, Cross RT, et al. Influenza hemagglutination-inhibition antibody titer as a correlate of vaccine-induced protection. J Infect Dis 2011;204:1879-1885. 


\section{Neurology \\ Neuroimmunology \& Neuroinflammation}

Impact of siponimod on vaccination response in a randomized, placebo-controlled study

Mike Ufer, Kasra Shakeri-Nejad, Anne Gardin, et al.

Neurol Neuroimmunol Neuroinflamm 2017;4;

DOI 10.1212/NXI.0000000000000398

This information is current as of September 14, 2017

Neurol Neuroimmunol Neuroinflamm is an official journal of the American Academy of Neurology.

Published since April 2014, it is an open-access, online-only, continuous publication journal. Copyright

Copyright $\odot 2017$ The Author(s). Published by Wolters Kluwer Health, Inc. on behalf of the American

Academy of Neurology.. All rights reserved. Online ISSN: 2332-7812.

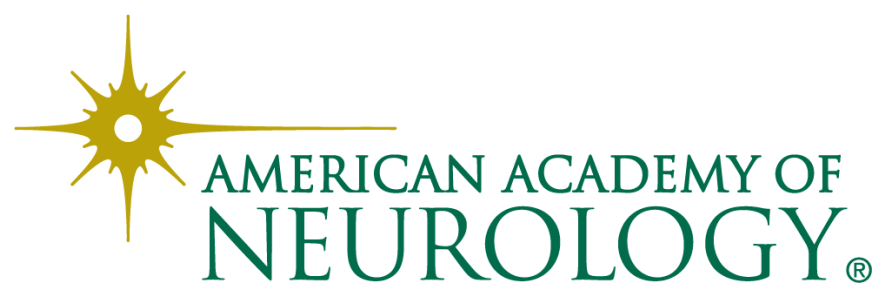




\section{Updated Information \& Services}

References

Citations

Subspecialty Collections

Permissions \& Licensing

Reprints including high resolution figures, can be found at: http://nn.neurology.org/content/4/6/e398.full.html

This article cites 27 articles, 1 of which you can access for free at: http://nn.neurology.org/content/4/6/e398.full.html\#\#ref-list-1

This article has been cited by 3 HighWire-hosted articles: http://nn.neurology.org/content/4/6/e398.full.html\#\#otherarticles

This article, along with others on similar topics, appears in the following collection(s):

All Clinical Neurology

http://nn.neurology.org//cgi/collection/all_clinical_neurology All Clinical trials

http://nn.neurology.org//cgi/collection/all_clinical_trials

All Immunology

http://nn.neurology.org//cgi/collection/all_immunology

Multiple sclerosis

http://nn.neurology.org//cgi/collection/multiple_sclerosis

Information about reproducing this article in parts (figures,tables) or in its entirety can be found online at:

http://nn.neurology.org/misc/about.xhtml\#permissions

Information about ordering reprints can be found online:

http://nn.neurology.org/misc/addir.xhtml\#reprintsus

Neurol Neuroimmunol Neuroinflamm is an official journal of the American Academy of Neurology.

Published since April 2014, it is an open-access, online-only, continuous publication journal. Copyright

Copyright $\odot 2017$ The Author(s). Published by Wolters Kluwer Health, Inc. on behalf of the American

Academy of Neurology.. All rights reserved. Online ISSN: 2332-7812.

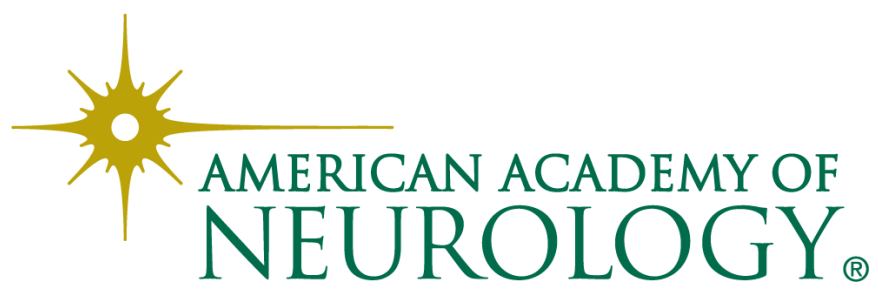

